\title{
Patient Satisfaction with General versus Local Anesthesia during Carotid Endarterectomy
}

\author{
Jan Mracek ${ }^{1} \quad$ Jakub Kletecka $^{2}$ Irena Holeckova $^{1} \quad$ Jiri Dostal $^{1} \quad$ Jolana Mrackova $^{3} \quad$ Jan Mork ${ }^{1}$ \\ Vladimir Priban ${ }^{1}$ \\ ${ }^{1}$ Department of Neurosurgery, Faculty of Medicine in Pilsen, Charles \\ University, University Hospital Pilsen - Pilsen, Czech Republic \\ 2 Department of Anesthesia and Intensive Care Medicine, Faculty of \\ Medicine in Pilsen, Charles University, University Hospital Pilsen - \\ Pilsen, Czech Republic \\ ${ }^{3}$ Department of Neurology, Faculty of Medicine in Pilsen, Charles \\ University, University Hospital Pilsen - Pilsen, Czech Republic \\ Address for correspondence Jan Mracek, MD, PhD, Department of \\ Neurosurgery, University Hospital Pilsen, Alej Svobody 80, \\ Pilsen 30460, Czech Republic \\ (e-mail: mracek@fnplzen.cz; janmracek1@seznam.cz). \\ J Neurol Surg A 2019;80:341-344.
}

\begin{abstract}
Background and Study Aims Both general and local anesthesia are used in our department for carotid endarterectomy (CEA). The decision as to which anesthetic technique to use during surgery is made individually. The aim of our study was to evaluate patient satisfaction and preference with the anesthesia type used.

Material and Methods The satisfaction of a group of 205 patients with regard to anesthesia used and their future preferences were evaluated prospectively through a questionnaire. The reasons for dissatisfaction were assessed.

Results CEA was performed under general anesthesia (GA) in 159 cases (77.6\%) and under local anesthesia (LA) in 46 cases (22.4\%). In the GA group, 148 patients (93.1\%) were satisfied; 30 patients $(65.2 \%)$ in the LA group were satisfied $(p<0.0001)$. The reason for dissatisfaction with GA were postoperative nausea and vomiting (7 patients), postoperative psychological alteration (3), and fear of GA (1). The reasons for dissatisfaction with LA were intraoperative pain (9 patients), intraoperative discomfort and stress (5), and intraoperative breathing problems (2). Of the GA group, 154 (96.9\%) patients would prefer $G A$ again, and of

Keywords

- carotid endarterectomy

- general anesthesia

- local anesthesia

- satisfaction

- patient the LA group, $28(60.9 \%)$ patients would prefer LA if operated on again $(p<0.0001)$. Overall, 172 patients $(83.9 \%)$ would prefer $G A$ in the future, and 33 patients $(16.1 \%)$ would prefer $L A$. Conclusion Overall patient satisfaction with CEA performed under both GA and LA is high. Nevertheless, in the GA group, patient satisfaction and future preference were significantly higher. Both GA and LA have advantages and disadvantages for CEA. An optimal approach is to make use of both anesthetic techniques based on their individual indications and patient preference.
\end{abstract}

\section{Introduction}

Carotid endarterectomy (CEA) is performed under general (GA) or local anesthesia (LA). Opinions on the benefit of both types of

(D)Jakub Kletecka's ORCID is https://orcid.org/0000-0003-04094916.

received

October 20, 2018

accepted after revision

February 16, 2019

published online

April 29, 2019

anesthesia have also long been a matter of controversy. It was not until the multicenter randomized GALA trial was published in 2008 that no difference in outcomes between GA and LA was found for carotid surgery. ${ }^{1}$ The results of the GALA trial also supported the Cochran review published in 2013. ${ }^{2}$ Both studies provided evidence suggesting that the anesthesiologist and surgeon should decide which anesthetic technique to use on an

(C) 2019 Georg Thieme Verlag KG Stuttgart · New York
DOI https://doi.org/ 10.1055/s-0039-1688692. ISSN 2193-6315. 
individual basis. Although both anesthetic procedures are considered equivalent, each presents its own advantages and disadvantages to both surgeon and anesthesiologist as well as to the patient. ${ }^{1-5}$ The need for a measure of patient satisfaction and preference regarding GA or LAduring CEA was discussed. ${ }^{6,7}$

Both GA and LA are used in our department, and the type of anesthesia is chosen on an individual basis. The aim of our work was to evaluate the satisfaction and preferences of patients for each type of anesthesia during CEA.

\section{Material and Methods}

A total of 205 patients underwent CEA under GA or LA (January 2012-December 2015). The type of anesthesia was selected on an individual basis by the neurosurgeon and anesthesiologist. Patient satisfaction with the type of anesthesia selected and future preferences were evaluated prospectively. The patients completed a simple questionnaire on the day of their discharge from the hospital consisting of two queries: 1 . Was I satisfied with the type of anesthesia? (A, satisfied; B, rather satisfied; C, rather dissatisfied; D, dissatisfied). 2. If I could choose next time, would I prefer surgery under general or local anesthesia? If patients were dissatisfied (C, D) with the type of anesthesia, they mentioned reasons for their dissatisfaction.

Statistical analysis was performed using SAS software (Cary, North Carolina, United States). The difference in the frequency of the investigated parameters between the given groups was tested using the Fisher exact test and the chisquare test for the specified proportion. Statistical significance was set at a level of $5 \%$.

GA was conducted as follows: total intravenous anesthesia, opiate premedication, induction of anesthesia (propofol $1.5-2 \mathrm{mg} / \mathrm{g}$, remifentanil $05-1 \mu \mathrm{g}$, and atracurium 0.5-0.6 $\mathrm{mg} / \mathrm{kg}$ ), tracheal intubation, and patients ventilated with a mixture of oxygen and nitrogen oxide (1:1). Anesthesia was maintained by continuous intravenous (IV) administration of $1 \%$ propofol $(3-10 \mathrm{mg} / \mathrm{kg} /$ hour $)$ with a repeated bolus of atracurium $(0.1-0.15 \mathrm{mg} / \mathrm{kg})$ and remifentanil $(0.5 \mu \mathrm{g} / \mathrm{kg})$.

Specifications of LA were as follows: opiate premedication, deep cervical plexus block (6-8 $\mathrm{mL} 0.75 \%$ ropivacaine) and superficial cervical plexus block (mixture of $5 \mathrm{~mL} 0.5 \%$ Chirocaine and 5-10 mL 1\% Mesocain), low doses of midazolam (0.5-1 mg IV) or alfentanil (0.25 mg IV), irrigation of the surgical field, and local infiltration with $1 \%$ Mesocain.

\section{Results}

CEA was performed under GA in 159 cases (77.6\%) and under LA in 46 cases (22.4\%). - Table 1 summarizes the demographic and perioperative data. No differences were noted in most of the variables between the GA and LA groups. There was a significantly higher proportion of symptomatic stenosis in the GA group and a significantly higher proportion of asymptomatic stenosis in the LA group $(p=0.0417)$. Postoperative wound hematoma was more common in the GA group $(p=0.0313)$. The average length of hospital stay was 6 days in the GA group and 4 days in the LA group, a statistically significant difference $(p<0.001)$.
Table 1 Demographic and perioperative data

\begin{tabular}{|c|c|c|c|}
\hline & $\begin{array}{l}\text { GA } \\
(n=159)\end{array}$ & $\begin{array}{l}\mathrm{LA} \\
(n=46)\end{array}$ & $p$ Value \\
\hline Age, y (mean) & $68(42-88)$ & $66(44-85)$ & NS \\
\hline Sex (\% male) & $116(73)$ & $32(70)$ & NS \\
\hline $\begin{array}{l}\text { Carotid stenosis, } \\
\% \text { (mean) }\end{array}$ & 75 (50-99) & 74 (50-99) & NS \\
\hline \multicolumn{4}{|l|}{ Symptoms (\%) } \\
\hline Asymptomatic & $14(9)$ & $9(20)$ & 0.0417 \\
\hline Symptomatic & $145(91)$ & $37(80)$ & 0.0417 \\
\hline \multicolumn{4}{|l|}{$\begin{array}{l}\text { Preoperative } \\
\text { clinical condition (\%) }\end{array}$} \\
\hline NIHSS 0 & $108(68)$ & $32(70)$ & NS \\
\hline NIHSS 1-3 & $51(32)$ & $14(30)$ & NS \\
\hline $\begin{array}{l}\text { Intraoperative } \\
\text { shunt (\%) }\end{array}$ & $5(3)$ & $1(2)$ & NS \\
\hline \multicolumn{4}{|l|}{ Complications (\%) } \\
\hline TIA & $2(1.3)$ & $1(2)$ & NS \\
\hline Stroke & $1(0.6)$ & $1(2)$ & NS \\
\hline Hyperperfusion & $1(0.6)$ & 0 & NS \\
\hline Internal & $2(1.2)$ & 0 & NS \\
\hline Nerve injuries & $9(6)$ & $3(6.5)$ & NS \\
\hline Wound hematoma & $8(5)$ & $1(2.2)$ & 0.0313 \\
\hline $\begin{array}{l}\text { Length of stay, } \\
\text { d (mean) }\end{array}$ & $6(3-14)$ & $4(2-8)$ & $<0.001$ \\
\hline
\end{tabular}

Abbreviations: GA, general anesthesia; LA, local anesthesia; NS, not significant; NIHSS, National Institute of Health Stroke Scale; TIA, transient ischemic attack.

In the GA group, 148 patients (93.1\%) were satisfied; 30 patients (65.2\%) in the LA group were satisfied (- Table 2 ). The difference in satisfaction is statistically significant $(p<0.0001)$. The reason for dissatisfaction with GA were postoperative nausea and vomiting ( 7 patients), postoperative psychological alteration (3), and fear of GA (1) (-Table 3). The reasons for dissatisfaction with LA were intraoperative pain (9 patients), intraoperative discomfort and stress (5), and intraoperative breathing problems (2) (-Table 4). Of the GA group, 154 (96.9\%) patients would prefer GA again, and of the LA group, 28 (60.9\%) patients would prefer LA if operated on again (-Table 2 ). The difference in preference is also statistically significant $(p<0.0001)$. When adding up preferences from both groups, a total of 172 patients (83.9\%) would prefer GA, and 33 patients (16.1\%) would prefer LA in the future (-Table 5 ).

Table 2 Satisfaction of patients with anesthesia type used and their future preference $(n=205)$

\begin{tabular}{|l|l|l|l|}
\hline & GA $(\boldsymbol{n}=159)$ & LA $(\boldsymbol{n}=\mathbf{4 6 )}$ & $\boldsymbol{p}$ value \\
\hline Satisfaction (\%) & $148(93.1)$ & $30(65.2)$ & $<0.0001$ \\
\hline Preference (\%) & $154(96.9)$ & $28(60.9)$ & $<0.0001$ \\
\hline
\end{tabular}

Abbreviations: GA, general anesthesia; LA, local anesthesia. 
Table 3 Reasons for dissatisfaction with general anesthesia $(n=159)$

\begin{tabular}{|l|l|}
\hline Postoperative nausea and vomiting & 7 patients \\
\hline Postoperative psychological alteration & 3 patients \\
\hline Fear of general anesthesia & 1 patient \\
\hline
\end{tabular}

Table 4 Reasons for dissatisfaction with local anesthesia $(n=46)$

\begin{tabular}{|l|l|}
\hline Intraoperative pain & 9 patients \\
\hline Intraoperative discomfort and stress & 5 patients \\
\hline Intraoperative breathing problems & 2 patients \\
\hline
\end{tabular}

Table 5 Overall future preference of anesthesia type $(n=205)$

\begin{tabular}{|l|l|l|}
\hline GA & LA & $p$ Value \\
\hline $172(83.9 \%)$ & $33(16.1 \%)$ & $<0.0001$ \\
\hline
\end{tabular}

Abbreviations: GA, general anesthesia; LA, local anesthesia.

\section{Discussion}

CEA can be performed under GA or LA. In most workplaces only one type of anesthesia is chosen according to their practice and tradition. ${ }^{1-5}$ We use both anesthetic techniques at our department. The type of anesthesia is always selected individually. The decision is made by the neurosurgeon and anesthesiologist. Each patient has a variety of risk factors that are reflected in the selection of the appropriate type of anesthesia.

We prefer GA for patients with a higher risk of intraoperative shunting (contralateral carotid occlusion, incomplete circle of Willis), which is safer in patients operated on under GA. We prefer GA in patients with unfavorable anatomical conditions (short thick neck, high position of carotid bifurcation, long or tandem carotid stenosis, ankylosing spondylitis) who would only tolerate surgery under LA with difficulties. GA is preferred for neurologically unstable and noncooperative patients (emergent CEA in patients with acute stroke). We also select GA when CEA is performed simultaneously with cardiac surgery. In patients taking clopidogrel (a significant number of symptomatic patients), local anesthesia (deep cervical plexus block) is contraindicated by our anesthesiologists, and therefore they are operated on under GA. ${ }^{8,9}$ Sleep apnea syndrome is one of the less common reasons for GA preference. ${ }^{10}$

The reasons for LA selection in our department are internal comorbidities, an unavailability of intraoperative electrophysiologic (EF) monitoring (technical difficulties, absence of electrophysiologist), and pacemaker (totally stimulated heart rhythm makes the assessment of intraoperative EF monitoring difficult and unreliable).

Although GA and LA are considered equivalent, patient satisfaction with the type of anesthesia used is not well known. ${ }^{6,7}$ Because we have long-term experience with both techniques of anesthesia during CEA, we decided to assess patient satisfaction and future preferences prospectively.
Demographic and perioperative data are summarized in -Table 1. The groups did not differ significantly in age, sex, the percentage significance of carotid artery stenosis, preoperative clinical condition, shunt insertion, or in most of the complications.

There was a significantly higher proportion of symptomatic stenosis in the GA group and thus a significantly higher proportion of asymptomatic stenosis in the LA group. Nevertheless, the statistical significance was weak $(p=0.0417)$. This difference is due to the preference of GA among patients taking clopidogrel.

The more frequent occurrence of postoperative hematoma in the GA group is probably associated with the use of clopidogrel $(p=0.0313)$. No patients taking clopidogrel were operated on under LA.

The average length of hospital stay was significantly longer in patients operated on under GA (6 days versus 4 days). The need for longer hospitalization after GA was confirmed in several studies. ${ }^{1,2,4-7}$ However, it was shown that longer hospital stay after GA was not considered a principal disadvantage by our patients. The shorter stay in our hospital is the major benefit of LA, particularly from an economic point of view.

Both groups were relatively homogeneous and differed in only a small number of demographic and perioperative variables. No correlation was found between satisfaction/ preference and any of the parameters in which the two groups differed significantly.

We found a significantly higher satisfaction of patients with GA (93.1\% versus 65.2\%). Dissatisfaction with GA (6.9\%) was mostly associated with its postoperative accompanying phenomenon. Nausea and vomiting and transient alteration of mental status were observed in the immediate postoperative period. One patient was afraid he would not wake up after surgery performed under GA. In contrast, the significantly higher dissatisfaction with LA (34.8\%) was associated with intraoperative difficulties: intraoperative pain, discomfort and stress, and breathing problems. We must emphasize that LA was performed by anesthesiologists who are skilled in locoregional anesthesia techniques. In cases where the patient felt pain, the neurosurgeon infiltrated the wound with a local anesthetic, and the anesthesiologist administered a low dose of IV alfentanil.

An even greater superiority was found for GA when assessing the preferences of patients if given the choice for a second hypothetical operation in the future. While $96.9 \%$ of the patients would choose GA again, LA would be preferred by only $60.9 \%$ of the patients in the future. This was a statistically significant difference $(p<0.0001)$. When adding up the preferences from both groups, a total of $83.9 \%$ of patients would prefer GA and $16.1 \%$ of patients would prefer LA in the future. It was shown that GA was undoubtedly more popular among our patients, and its position was strengthened even further after their experience with CEA.

Only a small number of articles have dealt with the satisfaction of patients with CEA, from the point of view of anesthetic technique. Moreover, most of these articles compared various techniques of locoregional anesthesia. ${ }^{11-13}$ Only two studies compared LA with GA. ${ }^{14,15}$ Unlike our report, neither of these studies detected significant differences in patient satisfaction 
between LA and GA. The overwhelming majority of patients were satisfied with both anesthetic techniques. In the report by McCarthy et al, operations under LA were associated with higher expectations of recovery. ${ }^{14}$

The data from our study provide evidence that most patients do not have an adverse experience with either GA or LA used for CEA. Nevertheless, our patients were more satisfied with GA and would prefer it in the future. In the context of the somewhat sporadic and, with regard to our findings, contradictory data in the literature, the superiority of GA in our study was rather surprising. That is why we were looking for an explanation for patient dissatisfaction with LA. One possible reason could be the deep cervical plexus block used by our anesthesiologists for LA. It is considered to be one of the most risky blocks associated with significant morbidity and therefore may be accompanied by patient dissatisfaction. ${ }^{16}$ In connection with the conclusions of our study, in our hospital we are gradually replacing the deep cervical plexus block by an intermediate cervical plexus block. The intermediate cervical plexus block should lead to lower morbidity, and its application with ultrasound control should be more accurate and more comfortable for patients. ${ }^{11,12,17}$

Our study has some limitations. The structure of the questionnaire was relatively simple and therefore did not always reflect in detail the degree of satisfaction. The smaller population of patients in the LA group could also be considered a limitation of the analysis. Our results could be partly influenced by the difference in the number of symptomatic and asymptomatic patients in the evaluated groups.

\section{Conclusion}

Overall patient satisfaction with CEA performed under both GA and LA was high. Nevertheless, patient satisfaction and future preference was significantly higher in the GA group. Administration of LA through the more invasive deep cervical plexus block may be the cause of patient dissatisfaction.

Both GA and LA have their advantages and disadvantages relative to CEA. An optimal approach is to make use of both anesthetic techniques based on their individual indications and patient preference.

\section{Funding}

This study was supported by the grant of Ministry of Health of the Czech Republic - Conceptual Development of Research Organization (Faculty Hospital in Pilsen [FNPI], 00669806).

\section{Conflict of Interest}

None declared.

\section{References}

1 Lewis SC, Warlow CP, Bodenham AR, et al; GALA Trial Collaborative Group. General anaesthesia versus local anaesthesia for carotid surgery (GALA): a multicentre, randomised controlled trial. Lancet 2008;372(9656):2132-2142

2 Vaniyapong T, Chongruksut W, Rerkasem K. Local versus general anaesthesia for carotid endarterectomy. Cochrane Database Syst Rev 2013;(12):CD000126

3 Dakour Aridi H, Paracha N, Nejim B, Locham S, Malas MB. Anesthetic type and hospital outcomes after carotid endarterectomy from the Vascular Quality Initiative database. J Vasc Surg 2018;67(05):1419-1428

4 Sideso E, Walton J, Handa A. General or local anesthesia for carotid endarterectomy-the "real-world" experience. Angiology 2011; 62(08):609-613

5 Ferrero E, Ferri M, Viazzo A, et al. Carotid endarterectomy: comparison between general and local anesthesia. Revision of our experience with 428 consecutive cases. Ann Vasc Surg 2010; 24(08):1034-1037

6 Tangkanakul C, Counsell CE, Warlow CP. Local versus general anaesthesia in carotid endarterectomy: a systematic review of the evidence. Eur J Vasc Endovasc Surg 1997;13(05):491-499

7 McCleary AJ, Maritati G, Gough MJ. Carotid endarterectomy; local or general anaesthesia? Eur J Vasc Endovasc Surg 2001;22(01):1-12

8 Horlocker TT, Wedel DJ, Rowlingson JC, et al. Regional anesthesia in the patient receiving antithrombotic or thrombolytic therapy: American Society of Regional Anesthesia and Pain Medicine Evidence-Based Guidelines (Third Edition). Reg Anesth Pain Med 2010;35(01):64-101

9 Working Party;Association of Anaesthetists of Great Britain \& Ireland; Obstetric Anaesthetists' Association; Regional Anaesthesia UK. Regional anaesthesia and patients with abnormalities of coagulation: the Association of Anaesthetists of Great Britain \& Ireland The Obstetric Anaesthetists' Association Regional Anaesthesia UK. Anaesthesia 2013;68(09):966-972

10 American Society of Anesthesiologists Task Force on Perioperative Management of patients with obstructive sleep apnea. Practice guidelines for the perioperative management of patients with obstructive sleep apnea: an updated report by the American Society of Anesthesiologists Task Force on Perioperative Management of patients with obstructive sleep apnea. Anesthesiology 2014;120(02):268-286

11 Alilet A, Petit P, Devaux B, et al. Ultrasound-guided intermediate cervical block versus superficial cervical block for carotid artery endarterectomy: The randomized-controlled CERVECHO trial. Anaesth Crit Care Pain Med 2017;36(02):91-95

12 Sait Kavaklı A, Kavrut Öztürk N, Umut Ayoğlu R, et al. Comparison of combined (deep and superficial) and intermediate cervical plexus block by use of ultrasound guidance for carotid endarterectomy. J Cardiothorac Vasc Anesth 2016;30(02):317-322

13 Martusevicius R, Swiatek F, Joergensen LG, Nielsen HB. Ultrasoundguided locoregional anaesthesia for carotid endarterectomy: a prospective observational study. Eur J Vasc Endovasc Surg 2012; 44(01):27-30

14 J McCarthy R, Trigg R, John C, Gough MJ, Horrocks M. Patient satisfaction for carotid endarterectomy performed under local anaesthesia. Eur J Vasc Endovasc Surg 2004;27(06):654-659

15 Quigley TM, Ryan WR, Morgan S. Patient satisfaction after carotid endarterectomy using a selective policy of local anesthesia. Am J Surg 2000;179(05):382-385

16 Pandit JJ, Bree S, Dillon P, Elcock D, McLaren ID, Crider B. A comparison of superficial versus combined (superficial and deep) cervical plexus block for carotid endarterectomy: a prospective, randomized study. Anesth Analg 2000;91(04):781-786

17 Barone M, Diemunsch P, Baldassarre E, et al. Carotid endarterectomy with intermediate cervical plexus block. Tex Heart Inst J 2010;37(03):297-300 